\title{
A Software Tool for Compressive Sensing based Time-Frequency Analysis
}

\author{
Andjela Draganić, Miloš Brajović, Irena Orović, Srdjan Stanković ${ }^{1}$ \\ ${ }^{1}$ University of Montenegro, Faculty of Electrical Engineering, Dzordza Vasingtona bb, 81000 Podgorica, Montenegro \\ andjelad@ac.me
}

\begin{abstract}
A software tool that implements Compressive sensing based time-frequency analysis and performs instantaneous frequency estimation, is proposed and described in the paper. A focus is made on the signals with fast varying instantaneous frequency (IF), which can be accurately estimated using complextime distribution. Therefore, the proposed tool offers different possibilities to adjust parameters of complex-lag distributions in order to comply with fast-varying IF laws. Moreover, beside the standard implementation based on the full set of samples, a compressive sensing based time-frequency approach is included in order to obtain sparse time-frequency representation. Sparse time-frequency representation is reconstructed from very few ambiguity domain observations. The tool performance is tested on real and synthetic signals.
\end{abstract}

Keywords -Compressive sensing, complex-time distribution, ambiguity domain, instantaneous frequency estimation, sparse representation

\section{INTRODUCTION}

Achieving highly concentrated representation in the timefrequency (TF) domain is one of the main requirements in TF based signal processing applications. A number of distributions is introduced to suit with different types of signals, such as the short-time Fourier transform (STFT), Wigner distribution (WD), S-method, etc. Particularly, for signals characterized by fast instantaneous frequency (IF) variations, the complex-lag distributions are employed in order to concentrate the energy around IF and to provide efficient TF representation [1]-[5]. Recently, it has been shown that the TF analysis may benefit from the Compressive sensing (CS) [6] and related signal reconstruction algorithms. Namely, the implementation of the complex-time distribution can be combined with the CS in the ambiguity domain aiming to provide very sparse $\mathrm{TF}$ representation. CS approach [4],[7],[8] defines conditions for high quality signal reconstruction in the cases of randomly under-sampled data, [7]-[10]. A signal representation needs to be sparse in certain domain, having zero values for most of the coefficients. Also, the signal should be dense in the domain of measurements acquisition. In this paper, we have observed the case of two-dimensional CS approach, where the measurements are collected in the ambiguity domain, while the reconstruction produces a sparse TF representations [9],[12][15]. The implementation of CS based type of TF analysis requires the knowledge of both the TF distributions and CS reconstruction using complex mathematical algorithms. Hence, in order to facilitate the calculation of $\mathrm{CS}$ based TF representations, the proposed software provides a user-friendly environment with different set up options. Particularly, the user can define the measurements acquisition process using the mask, chose the measurements inside or outside the mask, parameters of the complex-time distribution, etc. In order to evaluate its performance, several types of fast varying test signals are included within the tool. There is also a possibility to choose between analytic or real signals. Based on the calculation of the sparse TF distribution, the proposed software tool provides the estimation of the signal's IF. The estimation results are compared with the exact IF of the signal. The true and estimated IFs can be illustrated graphically within the tool, but can be also evaluated numerically using the mean square error. Consequently, the proposed software tool can be used for educational and research purposes, and could be upgraded for a specialized applications and scenarios.

The paper is organized as follows. Theoretical background on the distributions implemented within the software is given in Section II. The CS-based TF analysis is introduced in this section as well. Basic building blocks of the software tool and related functionalities are given in Section III. Section IV contains experimental results. Concluding remarks are given in the Section V.

\section{THEORETICAL BACKGROUND}

\section{A. Generalized complex-time distributions}

Accurate estimation of the signal's IF using TF distributions/representations is important for a number of realworld applications [4],[15],[16]. Various TF distributions have been developed and each of them is suitable for certain types of signals. In the case of fast varying IF, the commonly used distributions like the WD or Cohen class distributions fail to provide the satisfactory results regarding the concentration and ability to track the IF changes. Therefore, the higher order distributions with complex-lag argument are used for such signals. A general form of the complex-time distributions of order $N$ is given by [4]:

$$
T_{C T}^{N}(t, \omega)=\int_{-\infty}^{\infty} R_{N}(t, \tau) e^{-j \omega \tau} d \tau,
$$

where complex-lag moment $R_{N}(t, \tau)$ is defined as:

$$
R_{N}(t, \tau)=\prod_{k=0}^{N-1} x^{-\frac{j 2 \pi k}{N}}\left(t+\frac{\tau}{N} e^{\frac{j 2 \pi k}{N}}\right) .
$$

For the sake of simplicity, the $4^{\text {th }}$ order complex-time distribution $(N=4)$ is considered in the sequel, particularly its 
ambiguity domain-based implementation. The ambiguity functions with real and complex argument can be defined as:

$$
\begin{gathered}
A_{R}(\theta, \tau)=\int_{-\infty}^{\infty} x\left(t+\frac{\tau}{N}\right) x^{*}\left(t-\frac{\tau}{N}\right) e^{-j \theta t} d t, \\
A_{C}(\theta, \tau)=\int_{-\infty}^{\infty} R_{N}(t, \tau) e^{-j \theta t} d t,
\end{gathered}
$$

Both real-lag $A_{R}(\theta, \tau)$ and complex-lag $A_{C}(\theta, \tau)$ ambiguity functions can be filtered using the kernel $K(\theta, \tau)$ [4]:

$$
A_{C}^{K}(\theta, \tau)=K(\theta, \tau) A_{C}(\theta, \tau) ; A_{R}^{K}(\theta, \tau)=K(\theta, \tau) A_{R}(\theta, \tau) .
$$

The resulting ambiguity function can be obtained as a convolution of $A_{R}^{K}(\theta, \tau)$ and $A_{C}^{K}(\theta, \tau)$ within the window function $\zeta(\omega)$ :

$$
\begin{aligned}
& A_{C T}(\theta, \tau)=\int_{-\infty}^{\infty} \int_{-\infty}^{\infty} \int_{-\infty}^{\infty} \zeta(\omega) e^{-j \omega \tau_{1}} e^{j \omega\left(\tau-\tau_{1}\right)} \times \\
& \quad \times A_{R}^{K}\left(\theta_{1}, \tau_{1}\right) A_{C}^{K}\left(\theta-\theta_{1}, \tau-\tau_{1}\right) d \tau_{1} d \theta_{1} d \omega
\end{aligned} .
$$

Finally, the complex-time distribution is obtained as a twodimensional Fourier transform of $A_{C T}(\theta, \tau)$ :

$$
T_{C T}(t, \omega)=\frac{1}{2 \pi} \int_{-\infty}^{\infty} \int_{-\infty}^{\infty} A_{C T}(\theta, \tau) e^{j \theta t-j \omega \tau} d \tau d \theta .
$$

Choosing an appropriate kernel function $K(\theta, \tau)$ and distribution order $N$, the unwanted components such as cross terms or interference terms, can be removed from the TF plane, but usually on the cost of distribution concentration.

\section{B. CS-based TF analysis}

For a variety of signals in real applications, there is a certain domain in which the signal is represented by a few nonzero coefficients that bring important information. This property is referred as sparsity. Generally, the signals can be sparse either in time, frequency or time-frequency domain [17]-[20], and for such signals the sampling can be done with far less samples than it is required by the conventional sampling theorem. The sampling procedure should be done in a way which assures accurate signal reconstruction from the reduced set of samples. It has been proven that signal needs to be sampled in a random manner to provide satisfactory results. In this paper, we assume that a signal is sparse in TF domain, while it is acquired in the ambiguity domain. The measurements acquisition is done by randomly selecting the samples from the mask having low-pass characteristics (centered at the origin of ambiguity domain). Depending on the signal type, some measurement can be collected outside the mask, as well.

Starting from the relation between the ambiguity function and complex-time distribution:

$$
A(\theta, \tau)=\mathcal{F} \cdot T_{C T}(t, \omega),
$$

where $\mathcal{F}$ denotes 2D Fourier transform matrix, the 2D CS problem can be derived. Coefficients that belong to the signal terms are concentrated around the origin in the ambiguity plane, while the unwanted terms are dislocated. Hence, the measurements should be taken from the region around the origin, and then used to obtain a sparse TF distribution. The ambiguity function is multiplied by the mask:

$$
A_{m}(\theta, \tau)=\left[\begin{array}{ccc}
0_{P \times Q} & 0_{M_{x} \times Q} & 0_{P \times Q} \\
0_{P \times M_{y}} & 1_{M_{x} \times M_{y}} & 0_{P \times M_{y}} \\
0_{P \times Q} & 0_{M_{x} \times Q} & 0_{P \times Q}
\end{array}\right] A(\theta, \tau),
$$

and will be referred as the masked ambiguity function. where $M_{x}$ and $M_{y}$ denotes the rectangular mask dimensions, $P=(N-$ $\left.M_{x}\right), Q=\left(N-M_{y}\right) / 2$ and $N \times N$ is dimension of the ambiguity function.

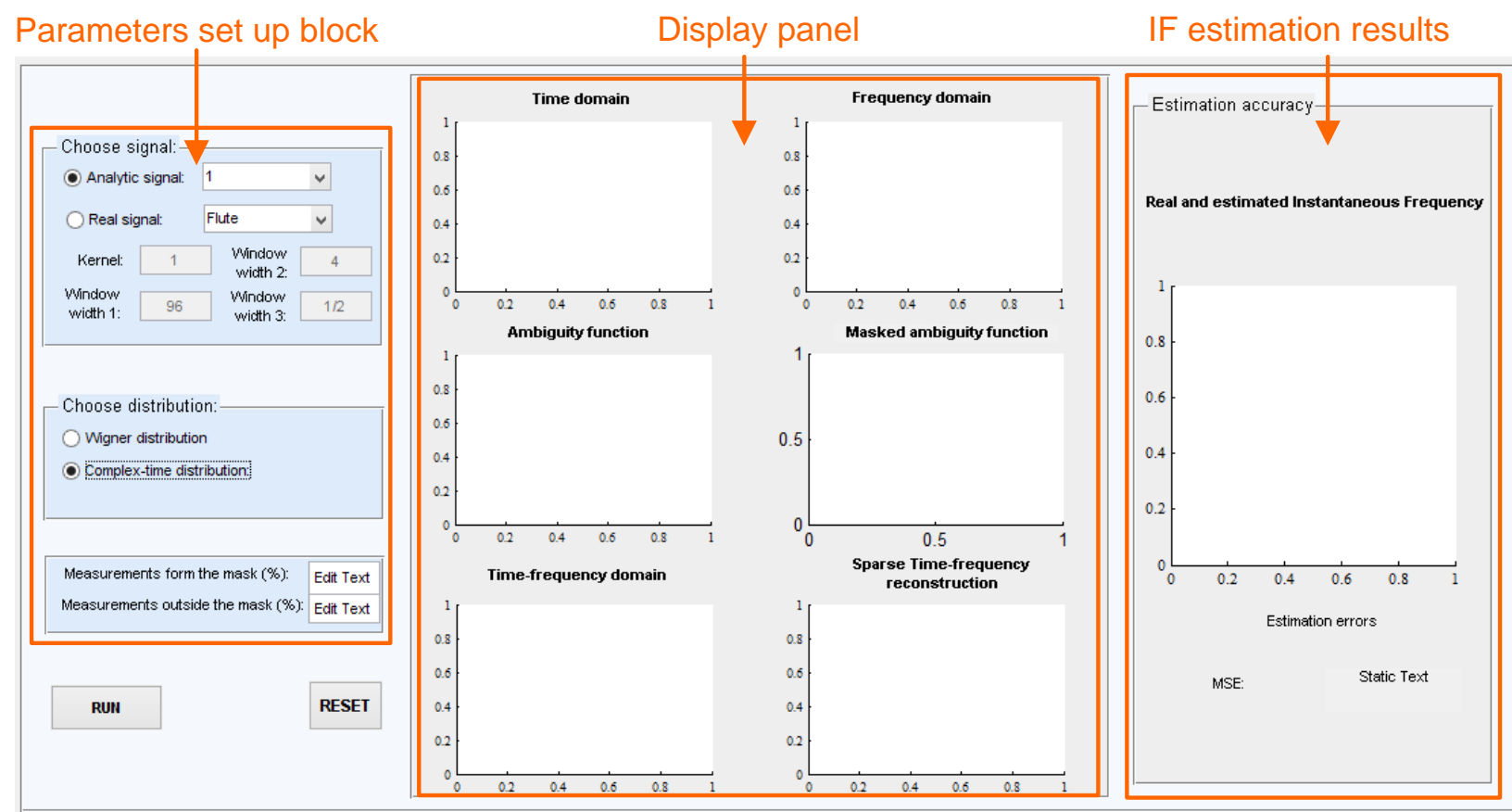

Figure 1. Main parts of the proposed software 
After the random selection of the ambiguity domain coefficients is performed, $A_{v e c}^{m}$ vector of the acquired ambiguity coefficients is obtained. The optimization problem is then formulated as follows:

$$
\min _{T_{s}}\left[\lambda\left\|T_{s}\right\|_{\ell_{1}}+\left(A^{m}-\mathcal{F}_{\text {part }} \cdot T_{s}\right) / 2\right]
$$

where $\mathcal{F}_{\text {part }}$ denotes subsampled 2D FT, keeping all columns of the matrix and only those rows whose positions corresponds to the positions of randomly selected samples. The solution of the above problem is an estimated sparse TF distribution $T_{s}$.

\section{THE PROPOSED SOFTWARE TOOL FOR CS-BASED TF ANALYSIS}

The software tool is consisted of several blocks:

- Parameters set up block - which is used to chose/define a signal, to specify the kernel and window parameters and to select appropriate distribution, and finally to specify the measurements selection;

- Display panel - used to represent the signal in the time and frequency domains, to display the original and masked ambiguity domain, $\mathrm{TF}$ and sparse $\mathrm{TF}$ representation;

- IF estimation results - containing graphical representation of the true and estimated IFs as well as the numerical evaluation using the mean square error.

The software tool is shown in Fig. 1. In Parameters set up block, a user can choose to test the CS-based IF estimation procedure on some of the already implemented synthetic signals, listed in the drop-down menu. Also, a real signal can be chosen as well, such as the real audio/musical signals for example. A window width parameters and the kernel type can be chosen within this part as well. Regarding the types of TF distributions, the WD and complex-time distribution are implemented within the tool. Since we have examined fast varying phase signals, the complex-time distribution is chosen in the examples.

In the part defining the measurements selection, one can choose certain amount of measurements within the mask, and certain amount of measurements outside the mask. In both cases, measurements are selected in a random manner. Mask is formed around the origin in ambiguity plane. Otherwise, the user can select a full set of samples in the ambiguity domain for the standard TF calculation procedure.

The block IF estimation results contains a graph for IF representation. Both, estimated and true IFs are displayed (using different colors - blue is for true IF while the red is for the estimated IF). Note that, the true IF in the cases of analytic signals will correspond to the first phase derivative, while in the cases of real signals, "true" IF is estimated from the starting, non-sparse TF distribution. In this part of the tool, the mean square error (MSE) between original and estimated IFs is shown as well.

\section{EXPERIMENTAL RESULTS}

The functionality of the software will be demonstrated on two types of signals: analytic and real one.

\section{Example 1: Flute signal (real signal case)}

Audio signals, such as piano, flute, guitar and clarinet signals produced by different instruments are included within the tool. When observing harmonics components, the small variations in the time-frequency domain can be noticed if the complextime distributions are used. Therefore, we have applied complex-time distributions and test the accuracy of the IF estimation from the CS-based sparse TF. The flute signal is considered for the experiment. When observing ambiguity function of this signal, it can be seen that components are not perfectly concentrated around the origin. Therefore, certain percent of measurements is used inside the mask $(10 \%)$ and outside the mask $(50 \%)$. The size of mask is $5 \times 5$, while the total size of the TF plane is $40 \times 40$ points.

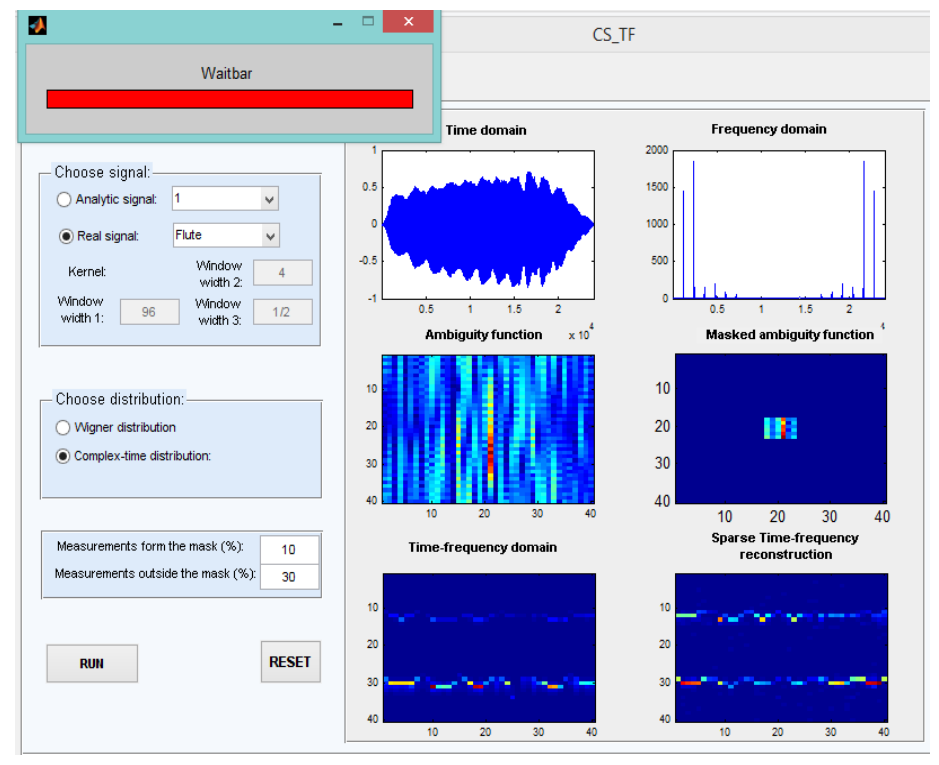

Figure 2. Sparse TF estimation for the flute signal



Figure 3. IFs for the components of the flute signal. Blue line is for true IFs, while red line denotes estimated Ifs 
The ambiguity function, the standard complex-time TF and CS-based sparse TF are shown in Fig. 2, for a considered flute signal (two harmonics are represented). The estimated IF of each component and corresponding MSEs are shown in Fig. 3.

\section{Example 2: Synthetic signal}

The results for sparse TF analysis and IF estimation, in the case of synthetic two-component signal, are shown in the sequel. Unlike the previous example, this signal has components concentrated around the origin in the ambiguity plane. Therefore, $50 \%$ percent of measurements is used only inside the mask. The size of mask is $25 \times 25$ (the total ambiguity plane size is $90 \times 90)$. Fig. 4 illustrates the signal in time, frequency and TF domain, as well as its sparse TF representation.

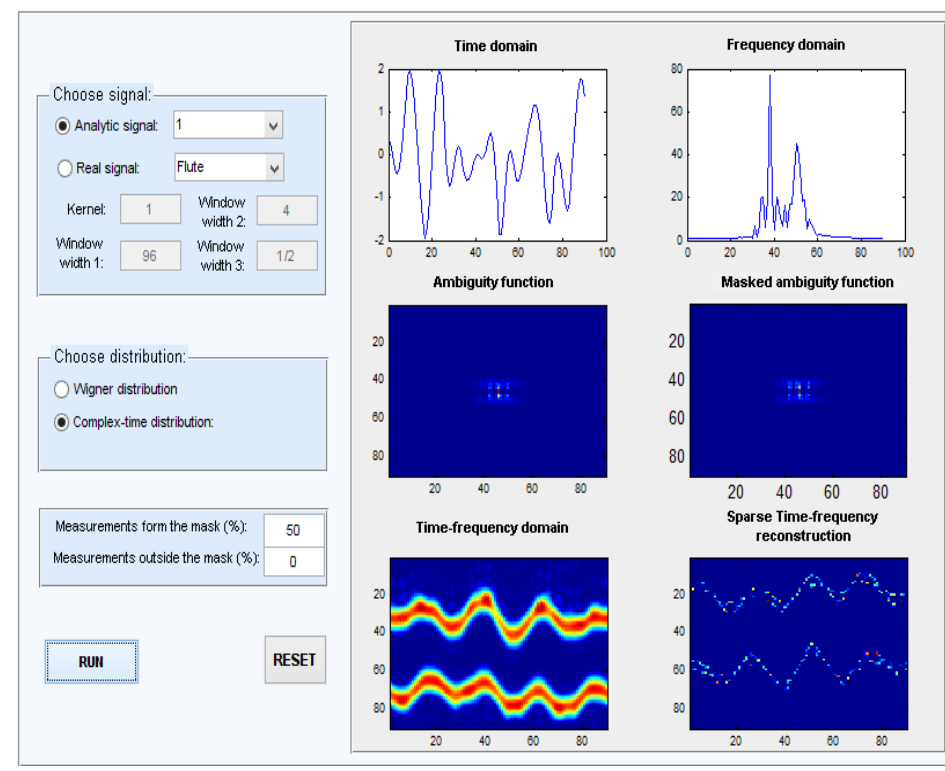

Figure 4. Results for obtaining sparse TF-analytic signal

\section{CONCLUSION}

In this paper we presented a software tool implementing the algorithms for obtaining sparse $\mathrm{TF}$ representation for IF estimation based on a reduced set of ambiguity domain measurements. Our focus was on the signals with fast varying IF, for which the standard distributions cannot provide satisfactory results. Therefore, we have implemented complextime distributions, both the standard and CS-based form, with the possibility to set up various parameters depending on the nature of test signals. This tool represents an initial version of the future multi-modular software - Virtual Instrument for Compressive Sensing. Hence, the future work will be oriented toward the extension of the software tool that will include more CS-based approaches, additional TF distributions, signal upload from a file format, and different mask definitions.

\section{ACKNOWLEDGMENT}

This work is supported by the Montenegrin Ministry of Science, project grant funded by the World Bank loan: CS-ICT "New ICT Compressive sensing based trends applied to: multimedia, biomedicine and communications".

\section{REFERENCES}

[1] S. Stankovic, I. Orovic, and C. Ioana, "Effects of Cauchy Integral Formula Discretization on the Precision of IF Estimation: Unified Approach to Complex-lag Distribution and its L-Form," IEEE Signal Processing Letters, vol. 16, No 4, pp. 307-310, 2009.

[2] S. Stanković, N. Zarić, I. Orović, and C. Ioana, "General form of timefrequency distribution with complex-lag argument, " Electronics Letters, vol. 44, No 11, pp. 699-701, 2008.

[3] R. Wang, and Y. Jiang, "ISAR Imaging of Ship Based on the Modified 4th Order Time-Frequency Distributions with Complex-Lag Argument," 2012 2nd RSETE conference, pp.1-4, 1-3 June 2012.

[4] I. Orović, M. Orlandić, S. Stanković, and Z. Uskoković, "A Virtual Instrument for Time-Frequency Analysis of Signals with Highly NonStationary Instantaneous Frequency," IEEE Transactions on Instrumentation and Measurements, vol. 60, No 3, pp. 791 - 803, 2011.

[5] S. Zukovic, M. Medenica, A. Draganic, I. Orovic, and S. Stankovic, "A Virtual Instrument for Compressive Sensing of Multimedia Signals," 56th International Symposium ELMAR 2014, Zadar, Croatia, 2014.

[6] E. J. Candès, and M. B. Wakin, "An Introduction To Compressive Sampling, " IEEE Signal Processing Magazine 2008, vol 25, No 2, pp. 21-30, 2008.

[7] D. Donoho, "Compressed sensing," IEEE Transaction on Information Theory, vol. 52, No. 4, pp. 1289 - 1306, 2006

[8] S. Stankovic, I. Orovic, and LJ. Stankovic, "An Automated Signal Reconstruction Method based on Analysis of Compressive Sensed Signals in Noisy Environment," Signal Processing, vol. 104, pp. 43-50, November 2014.

[9] P. Boyd, and L. Vandenberghe, Convex Optimization. Cambridge University Press, Mar 8, 2004 - Business \& Economics - 716 pages.

[10] J. A. Tropp, "Greed is good: algorithmic results for sparse approximation," IEEE Transactions on Information Theory, vol. 50, No.10, pp. 2231-2242, October 2004.

[11] P. Flandrin, and P. Borgnat, "Time-Frequency Energy Distributions Meet Compressed Sensing," IEEE Transactions on Signal Processing, vol.58, no.6, pp.2974, 2982, June 2010.

[12] A. Jung, G. Taubock, and F. Hlawatsch, "Compressive nonstationary spectral estimation using parsimonious random sampling of the ambiguity function," IEEE/SP 15th Workshop on Statistical Signal Processing, SSP '09., pp.642-645, 31 August 2009- 3 September 2009.

[13] P. Borgnat, and P. Flandrin, "Time-frequency localization from sparsity constraints," IEEE International Conference on Acoustics, Speech and Signal Proceesing ICASSP-08, pp. 3785-3788, Las Vegas (NV), 2008.

[14] I. Orovic, S. Stankovic, and M. Amin, "Compressive Sensing for Sparse Time-Frequency Representation of Nonstationary Signals in the Presence of Impulsive Noise," SPIE Defense, vol 8717, United States, 2013.

[15] I. Orovic, and S. Stankovic, "A Class of Highly Concentrated TimeFrequency Distributions Based on the Ambiguity Domain Representation and Complex-Lag Moment," EURASIP Journal on Advances in Signal Processing, vol. 2009, Article ID 935314, 9 pages.

[16] B. Boashash, "Estimating and Interpreting The Instantaneous Frequency of a Signal - Part 1: Fundamentals", Proceedings of the IEEE, vol. 8, No 4, pp. 520-538, 1992.

[17] I. Orovic, S. Stankovic, and T. Thayaparan, "Time-Frequency Based Instantaneous Frequency Estimation of Sparse Signals from an Incomplete Set of Samples," IET Signal Processing, Special issue on 
Compressive Sensing and Robust Transforms, vol. 8, No 3, pp. 239 245, May, 2014.

[18] I. Orovic, and S. Stankovic, "Improved Higher Order Robust Distributions based on Compressive Sensing Reconstruction," IET Signal Processing, vol. 8, No 7, pp. 738 - 748, September 2014.

[19] D. Angelosante, G.B. Giannakis, and E. Grossi, "Compressed sensing of time-varying signals," 16th International Conference on Digital Signal Processing, pp.1-8, July 2009.
[20] S. Stankovic, LJ. Stankovic, and I. Orovic, "Relationship between the Robust Statistics Theory and Sparse Compressive Sensed Signals Reconstruction," IET Signal Processing, Special issue on Compressive Sensing and Robust Transforms, vol. 8, No 3, pp. 223-229, May, 2014. 